OPEN ACCESS

Edited by:

Leonardo Freire-de-Lima, Federal University of Rio de Janeiro,

Brazil

Reviewed by:

Michael R. King,

Cornell University, USA

Leonardo Marques Da Fonseca, Federal University of Rio de Janeiro,

Brazil

${ }^{*}$ Correspondence:

Michael E. O'Dwyer

michael.odwyer@nuigalway.ie

Specialty section:

This article was submitted to Molecular and Cellular Oncology, a section of the journal Frontiers in Oncology

Received: 22 February 2016 Accepted: 30 March 2016 Published: 18 April 2016

Citation:

Natoni A, Macauley MS and O'Dwyer ME (2016) Targeting Selectins and Their Ligands in

Cancer.

Front. Oncol. 6:93. doi: 10.3389/fonc.2016.00093

\section{Targeting Selectins and Their Ligands in Cancer}

\author{
Alessandro Natoni ${ }^{1}$, Matthew S. Macauley ${ }^{2}$ and Michael E. O'Dwyer ${ }^{1,3 *}$ \\ ${ }^{1}$ Biomedical Sciences, National University of Ireland Galway, Galway, Ireland, ${ }^{2}$ Department of Chemical Physiology, The \\ Scripps Research Institute, La Jolla, CA, USA, ${ }^{3}$ School of Medicine, National University of Ireland Galway, Galway, Ireland
}

Aberrant glycosylation is a hallmark of cancer cells with increased evidence pointing to a role in tumor progression. In particular, aberrant sialylation of glycoproteins and glycolipids has been linked to increased immune cell evasion, drug evasion, drug resistance, tumor invasiveness, and vascular dissemination, leading to metastases. Hypersialylation of cancer cells is largely the result of overexpression of sialyltransferases (STs). Differentially, humans express twenty different STs in a tissue-specific manner, each of which catalyzes the attachment of sialic acids via different glycosidic linkages ( $\alpha 2-3$, $\alpha 2-6$, or $\alpha 2-8$ ) to the underlying glycan chain. One important mechanism whereby overexpression of STs contributes to an enhanced metastatic phenotype is via the generation of selectin ligands. Selectin ligand function requires the expression of sialyl-Lewis $X$ and its structural isomer sialyl-Lewis $\mathrm{A}$, which are synthesized by the combined action of alpha $\alpha 1$-3-fucosyltransferases, $\alpha 2-3$-sialyltransferases, $\beta 1$-4-galactosyltranferases, and $N$-acetyl- $\beta$-glucosaminyltransferases. The $\alpha 2-3$-sialyltransferases ST3Gal4 and ST3Gal6 are critical to the generation of functional $\mathrm{E}$ - and P-selectin ligands and overexpression of these STs have been linked to increased risk of metastatic disease in solid tumors and poor outcome in multiple myeloma. Thus, targeting selectins and their ligands as well as the enzymes involved in their generation, in particular STs, could be beneficial to many cancer patients. Potential strategies include ST inhibition and the use of selectin antagonists, such as glycomimetic drugs and antibodies. Here, we review ongoing efforts to optimize the potency and selectivity of ST inhibitors, including the potential for targeted delivery approaches, as well as evaluate the potential utility of selectin inhibitors, which are now in early clinical development.

Keywords: sialyltransferase, metastasis, niche, sialic acid, glycosylation, selectin, glycomimetic, tumor

\section{INTRODUCTION}

It is well established that one of the most frequent changes in cancer cells is the pattern of cell surface glycosylation $(1,2)$. Because glycans present on the plasma membrane influence the ability of cells to interact with their surrounding microenvironment, altered glycosylation enables cancer cells to acquire specific capabilities to interact with all components of the microenvironment, such as growth factors, chemokines, extracellular matrix, and cell to cell contact. As a consequence, processes such as adhesion, mobilization, and migration are also altered in cancer (3). The glycosylation pattern of cancer cells may also change during disease progression; indeed, metastatic cancer cells display profound differences in cell surface glycosylation, compared not only to the normal cells but also to 
the original tumor (4-7). Changes in glycans on cancer cells often arise from a combination of mechanisms, such as overexpression or downregulation of the glycan's protein or lipid scaffold or modifications of the metabolic pathways responsible for the generation of specific glycans (1).

One of such changes in the glycome of cancer cells is the increased presence of sialic acid sugars on the surface of cancerous cells (8). Hypersialylation of tumor cells has been associated with a metastatic phenotype and inferior outcome in patients with cancer (9). Sialic acids represent a group of sugars based on the neuraminic acid scaffold, with the most frequent being $\mathrm{N}$-acetylneuraminic acid (Neu5Ac; Figure 1A). Sialic acids are predominantly found at the non-reducing termini of $\mathrm{N}$ - and $\mathrm{O}$-linked glycans attached to proteins or on glycolipids. Sialylation of glycans is carried out by a complex, yet highly specific, series of enzymatic processes that take place in the ERGolgi apparatus, and are responsible for the covalent linkage of sialic acids to galactose (Gal), $N$-acetylgalactosamine (GalNAc), $\mathrm{N}$-acetylglucosamine (GlcNAc), or to another sialic acid (polysialic acids). These enzymatic reactions are performed by a class of glycosyltranferases termed the sialyltransferases (STs).

\section{THE SIALYLTRANSFERASE FAMILY}

The ST family is part of the larger glycosyltransferase superfamily generally expressed in the secretory pathway. Recently, there has been a surge in structural understanding of the family, knowledge

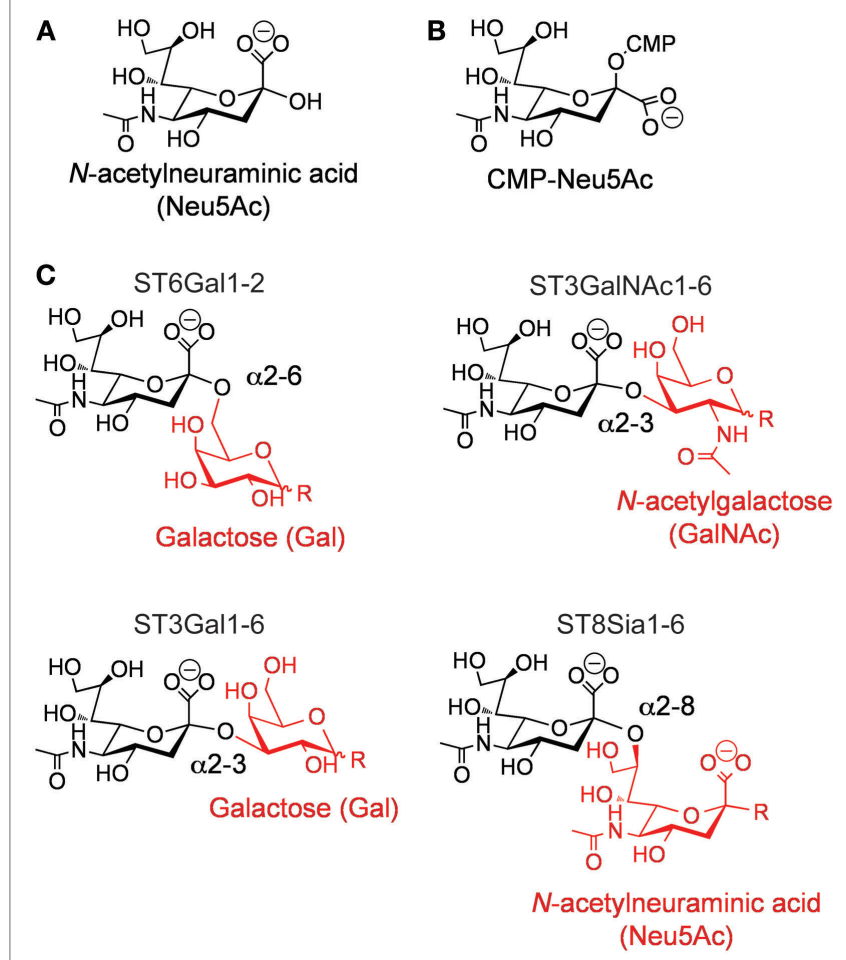

FIGURE 1 | (A) Structure of $N$-acetylneuraminic acid. (B) Structure of cytidine-5'-monophospho-(CMP)-sialic acid. (C) Types of sialic acid linkages catalyzed by the different members of the mammalian ST family. of which members is involved in biosynthesis of selectin ligands under both healthy and cancerous conditions, and advances in the ability to inhibit STs with a cell permeable inhibitor.

\section{Overview of the ST Family}

In humans, the ST family is composed of 20 individual members (10-12). These enzymes are expressed in a cell- and tissuespecific manner, proving each cell type with a unique "sialome" (13). All mammalian STs use cytidine-5' -monophospho-(CMP)sialic acid (Figure 1B) as their donor substrate and a glycan as their receptor. Exquisite stereoselectivity results in ST-catalyzed formation of an $\alpha$-linked sialic acid to a precise hydroxyl group on a specific particular saccharide residue. The combination of the acceptor saccharide residue and the precise hydroxyl group on this residue can be used to divide the family into four distinct families (Figure 1C) $(11,12,14)$. For example, ST6Gal enzymes catalyze the transfer of sialic acid to the $6^{\prime}$-hydroxyl group of a Gal residue, ST3Gal enzymes catalyze the transfer of sialic acid to the 3 '-hydroxyl group of a Gal residue, ST3GalNAc enzymes catalyze the transfer of sialic acid to the $6^{\prime}$-hydroxyl group of a GalNAc, and ST3Sia enzymes catalyze the transfer of sialic acid to the $8^{\prime}$-hydroxyl group of another sialic acid residue.

All mammalian STs belong to glycosyltransferase family 29 (GT29) in the CAZy database (www.cazy.org). Only recently, the first structures of mammalian STs have been solved. The first was ST3Gal1 from porcine (15), which is the enzyme that masks the peanut agglutinin (PNA) ligand (Gal $\beta 1-3 \mathrm{GalNAc}$ ) on complex $O$-glycans. The structure of ST3Gall showed for the first time how active site residues position the appropriate hydroxyl group on the acceptor for nucleophilic attack. Structures of both rat and human ST6Gal1 were also recently solved $(16,17)$. Fortuitously, the complex $N$-glycan on a neighboring dimeric unit was trapped in the active site of the human enzyme, providing structural insight into the well described preference of this enzyme for the $\alpha 1-3$ mannose branch on complex $N$-glycans. Further structural insight into this sub-family of ST awaits a cocrystal structure in which electron density of sialic acid is clearly observed. More recently, the structure of ST8Sia3 was determined, which has led to insight into how this sub-family uses a positively charged groove to position the extended polysialic acid oligosaccharide acceptor (18). Together, the structures of the first three mammalian STs provide an excellent starting point for understanding the structure basis for catalysis by the mammalian ST family. Nevertheless, there is much more work to be done in determining how different the active site architecture is between the different STs; based on differences observed in the three structures solved to date, it seems reasonable to speculate that, although challenge, it may be possible to exploit differences between members of the family to ultimately develop selective inhibitors that target individual sub-classes, or even members, of the ST family.

\section{Sialyltransferase Overexpression in Cancer}

Many of the STs are overexpressed in various forms of cancer, which has been reviewed in detail elsewhere (19-24). In many cases, this overexpression has been correlated with cell surface 
overexpression of the product for that particular ST. For example, many cancer cells overexpress ST6Gal1, and as a consequence, stain strongly with the Sambucus nigra (SNA) lectin that recognizes sialic acid residues $\alpha 2-6$-linked to Gal (22). In another example, gliomas were shown to express ST3Gal1, which correlated with low staining of cells and tumors by PNA (25). On the other hand, several reports have also documented that loss or downregulation of ST expression correlates with cancer progression $(26,27)$. These findings highlight the need for careful studies that systematically modulate ST expression and activity to determine whether it is simply aberrant glycosylation or specifically hypersialylation that plays a role in tumor progression.

Overexpression of STs and the resultant hypersialylation in cancer has been implicated in many stages of tumorigenesis ( 7 , $20,21,24)$. Studies have documented the roles for hypersialylation in drug and radiation resistance $(28,29)$. Recent work has found that hypersialylation is also involved in evasion from the immune system, with numerous types of cancer cells expressing high levels of sialylated ligands of the inhibitory receptors sialic acidbinding, immunoglobulin-like lectin-(Siglec)-7 and Siglec-9, which in turn recruit these Siglecs to inhibit natural killer (NK) cell killing $(30,31)$ or neutrophil activation (32). Hypersialylation is also implicated in enhancing tumor invasiveness by enhancing cellular proliferation and motility through constitutive activation of pathways involved in cell growth and motility $(33,34)$. A critical role for hypersialylation in cancer metastasis has also been suggested for certain types of cancer. For example, sialylated ligands of the Selectin family of adhesion proteins ligands have been described on multiple myeloma (MM) cells $(35,36)$ and breast cancer cells (37) and have been shown to be critical for homing and metastasis of these cancer cells. Similar observations are suggested based on correlative studies in renal cell carcinoma (38) and lung cancer (39). Based on the broad therapeutic interest around preventing cancer metastasis, this aspect is described in more detail in the following section.

\section{SELECTIN AND THEIR LIGANDS IN CANCER METASTASIS}

Sialic acids are incorporated within many different carbohydrate structures, including sialyl Lewis $\mathrm{X}\left(\mathrm{SLe}^{\mathrm{x}}\right)$ and its isomer sialyl Lewis A (SLe ; Figure 2). These tetrasaccharide structures are composed of $\alpha 2$-3-linked sialic acid on the GlcNAc backbone. $\mathrm{SLe}^{\mathrm{x}}$ and SLe $\mathrm{e}^{\mathrm{a}}$ represent the minimal recognition motif for ligands of selectins, a family of lectins whose functions are well characterized as mediators of leukocytes trafficking $(40,41)$. Three types of selectins have been described so far, the L-, E-, and P-selectins. Selectins are type I membrane proteins composed of a $\mathrm{N}$-terminus C-type lectin domain followed by an epidermal growth factor (EGF)-like motif, a series of consensus repeats, a transmembrane domain, and a short cytoplasmatic tail. By interacting with SLe ${ }^{\mathrm{x}}$ and SLe ${ }^{\mathrm{a}}$ containing glycoproteins and glycolipids, selectins are responsible for the slow tethering and rolling of leukocytes on the vascular endothelium that is the first step of leukocytes extravasation during inflammation or lymphocytes homing. As is often seen during oncogenic transformation, cancer cells take advantage of this physiological process to spread and colonize to distant organs during the metastatic cascade $(3,42)$. Indeed, extravasation of tumor cells during metastasis is the best documented function of selectins and their ligands in cancer (43-45). However, recent evidence suggests a role of selectins/selectin ligands interactions beyond the extravasation process, such as emboli formation, formation of a permissive microenvironment for metastasis, and retention of tumor cells in protective niches.

\section{Selectins and Their Ligands during Extravasation and Homing of Cancer Cells}

Selectins have been directly implicated in tumor extravasation for their ability to support tumor cell rolling on activated endothelium in a process that mirrors leukocytes extravasation. In a small cell lung cancer xenograft model, it has been shown that cancer cells rolled in vivo on tumor necrosis factor $\alpha$-(TNF $\alpha-)$ treated vessels, although the rolling velocity was seven times faster than that of leukocytes (46). Moreover, when cancer cells were transplanted in E- and P-selectin knockout (KO) mice, the number of metastases was significantly lower than wild-type (WT) mice, demonstrating the importance of selectins in metastases. The capability of a particular tumor to extravasate and therefore metastasize might correlate with the expression levels of selectin ligands. In colon carcinoma, there was a direct association between the levels of E-selectin ligands and the metastatic phenotype (47). Colorectal cancer cells expressing high levels of SLe extravasate more efficiently than cancer cells with low SLe in vivo. Similarly, the highly metastatic breast cancer cell line ZR-75-1 establishes stronger interactions with E-selectin in vitro compared to the less metastatic cell line MCF7 (48). Curiously, Mucin-1 (MUC1) serves as the E-selectin ligand in these cells but also enables firm adhesion by binding to intracellular adhesion molecule- 1 (ICAM-1) suggesting that selectin scaffolds may have more than one role during tumor extravasation. Mouse prostate cancer cells engineered to overexpress fucosyltransferase (FUT)3, 6, and 7, show different ability to roll on E-selectin in vitro with the FUT6-overexpressing cells displaying the highest number of rolling cells (49). Accordantly, the FUT6-overexpressing cells exhibit the highest metastatic potential in the bone compared to FUT3 and FUT7-overexpressing cells. Recently, we have shown that MM cells overexpress ST3Gal6 and have high levels of $\alpha 2-3$ linked sialic acid on the cell surface, suggesting a role of selectin ligands in homing of MM to the bone marrow (36). Knocking down ST3Gal6 decreased the levels of $\alpha 2$-3-linked sialic acid, impaired transendothelial migration of myeloma cell in vitro, and importantly reduced homing in vivo, indicating that the levels of selectin ligands influences myeloma trafficking into the bone marrow niche.

The ability of tumor cells to interact with selectins has been proposed to correlate to some extent with disease progression. In MM, we observed that the expression levels of ST3Gal6 increased with disease progression (36). Moreover, the P-selectin glycoprotein ligand-1 (PSGL-1) gene expression was found to increase with disease progression and, more recently, the percentage of potential E-selectin ligands bearing primary myeloma cells was shown to be higher in relapsed versus diagnosed patients $(50,51)$. 
A

$$
\text { Sle } e^{x}
$$

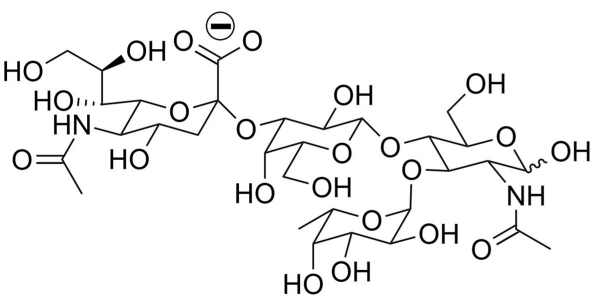

B $\quad \mathrm{Sle}^{\mathrm{a}}$

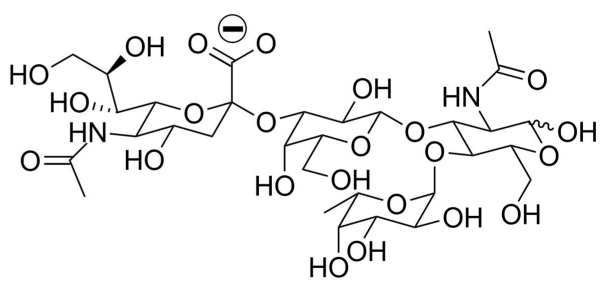

GIcNAc

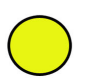

Gal

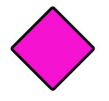

Sia

Sialic Acid

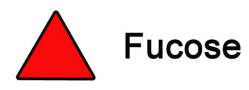

FIGURE 2 | Structure of SLe ${ }^{x}(A)$ and its structural isomer SLe ${ }^{a}(B)$

Similarly, in acute myeloid leukemia (AML), the expression of E-selectin ligands is higher in relapsed compared to newly diagnosed patients (52). In prostate cancer, the ex vivo interactions between circulating tumor cells (CTC) isolated from patients and E-selectin on activated endothelial cells correlated with the clinical response of castration resistant prostate cancer patients with no interactions during clinical response and many interactions at time of tumor progression (53). These data suggest a dynamic modulation of selectin ligands expression as the disease develops to a more aggressive phenotype.

Expression of selectins may also dictate to some extent tissue tropism for metastatic cancer cells. Transgenic mice overexpressing E-selectin in the liver have the propensity to develop hepatic metastasis when injected with melanoma cells expressing E-selectin ligands (54). In this system, murine melanoma cells that naturally metastasize to the lungs were engineered to express E-selectin ligands and injected in WT or transgenic mice with ubiquitously or liver specific expression of E-selectin. Liver metastases were observed only in E-selectin transgenic mice. The prevalence of liver metastasis, even in mice where E-selectin was ubiquitously expressed at similar levels in all tissues, indicates that additional factors influence the metastatic process. The large vasculature in combination with low shear stress typical of the liver may provide tumor cells with more potential adhesive sites than other organs. E-selectin dependency was also demonstrated in vivo for spontaneous breast metastasis to the lung and in a murine model of spontaneous liver metastasis $(55,56)$. In both studies, expression of E-selectin in the vasculature was dependent on pro-inflammatory and pro-angiogenic cytokines induced or even released by tumor cells, suggesting that primary tumors may promote an inflammatory activation of the endothelium in distant organs to facilitate metastatic seeding $(56,57)$. Selectins were also involved in engraftment of chronic myelogenous leukemia (CML) and chronic eosinophilic leukemia (CEL) in a severe combined immunodeficiency (SCID) mouse xenograft model (58). $\mathrm{E}$ - and P-selectin KO mice that were injected with leukemic cells displayed remarkably increased survival and little organ infiltration compared to WT mice. Moreover, the murine breakpoint cluster region-Abelson 1 positive $\left(\mathrm{BRC}-\mathrm{ABL}^{+}\right)$progenitor cells that resemble human CML demonstrated low engraftment in recipient mice deficient for E-selectin (59). Murine BRC-ABL1 ${ }^{+}$ progenitor cells deficient either in PSGL-1, CD44, and FUT4, 7 or core $2 \beta 1-6-N$-acetylglucosaminyl-transferase (core2 GlcNAcT-I) were impaired for engraftment.

Selectins other than E-selectin are also implicated in tumor extravasation and homing. In the previous study, L-selectin was also shown to be important for BRC-ABL1+ progenitor cells homing in the bone marrow (59). Recently, L-selectin has been shown to be one of the key receptors for chronic lymphocytic leukemia (CLL) homing and extravasation into the lymph nodes, an important niche where CLL cells proliferate and become resistant to chemotherapy (60). L-selectin was also shown to facilitate tumor metastases by recruiting leukocytes at sites of tumor embolization (61). Leukocytes may assist extravasation by bridging tumor 
cells or thrombi to the vascular endothelium and breaching the endothelial barrier. Indeed, E-selectin is frequently upregulated in the endothelium of the metastatic environment (62). Since L-selectin is an E-selectin ligand $(63,64)$, E-selectin present on the metastatic endothelium may contribute to leukocyte recruitment and establishment of a metastatic niche. Recently, the expression of PSGL-1 on monocytes has been shown to promote tumor extravasation (65). Although the type of selectin binding to PSGL-1 was not determined, this study further emphasizes the important role of selectins/selectin ligands interaction in tumor extravasation. P-selectin was found to be important in a metastatic model of melanoma and breast cancer (66). In this study, B16F1 melanoma cells were able to metastasize in the lungs and liver of NK-depleted/deficient mice in a P-selectin-dependent manner. Intriguingly, while metastases in the lungs were dependent on P-selectin expression on platelets and endothelial cells, hepatic metastases relied only on endothelial P-selectin. From these and other studies, it is clear that selectins and their ligands participate in the extravasation of cancer cells and they also contribute, together with other factors, to the site-specific colonization of metastatic cells.

Cancer cells exhibit a metabolic shift from oxidative to anaerobic glycolysis, this is known as the Warburg effect, which corresponds to increased gene expression of sugar transporters and glycolytic enzymes in cancer cells and is part of the adaptation of cancer cells to a hypoxic tumor microenvironment. These changes have been recently linked to induction of genes related to the expression of SLe ${ }^{\mathrm{x}}$ in cancer (67). This includes ST3Gal1, FUT7, and uridine 5' diphosphate (UDP)-Gal transporter-1 (UGT1), which are induced when colon cancer cells are grown under hypoxic conditions; interestingly, this is believed to be mediated by hypoxia inducible factor (HIF) (68). This leads to higher expression of SLe ${ }^{\mathrm{x}}$ and SLe $\mathrm{S}^{\mathrm{a}}$ on cancer cells and is likely to at least partially explain the increased SLe ${ }^{\mathrm{x}}$ - determinant expression seen in some cancers, which may be accompanied by a concomitant increase in E-selectin binding activity. We have made a similar observation in MM. When grown in culture under hypoxic conditions $\left(1 \% \mathrm{O}_{2}\right)$, we have seen marked upregulation of ST3Gal6 transcripts along with a significant increase in E-selectin ligand expression (69). This process refers to the "neosynthesis" hypothesis related to the mechanism of enhanced expression of carbohydrate determinants of selectin ligands in cancers $(70,71)$. This was further examined in a study looking at the association between SLe ${ }^{\mathrm{x}}$ and SLe ${ }^{\mathrm{a}}$ expression on colon cancer cells and epithelial-mesenchymal transition (EMT). Induction of EMT was shown to increase SLe ${ }^{\mathrm{x}}$ and SLe ${ }^{\mathrm{a}}$ expression and enhance E-selectin binding. In this study, transcript levels of ST3Gal1/3/4 and FUT3 were significantly elevated and found to be regulated by c-Myc. This study outlines the role of SLe ${ }^{\mathrm{x}}$ and SLe ${ }^{\mathrm{a}}$ expression in mediating selectin binding during EMT (72). It is thus possible that hypoxia and other drivers of EMT may lead to the mobilization of selectin ligand-bearing cells that are then capable of homing to distant sites where selectins are expressed.

\section{Selectins and Tumor Emboli Formation}

The first association between cancer and thrombosis, and subsequent microemboli formations, came from Trousseau in 1895 , and since then, this association was further established by a large body of clinical evidence (73). Indeed, cancer patients often experience malignancy-associated thrombosis, which is the second commonest cause of cancer mortality (74). In cancer patients and in particular in metastatic cancer patients, the risk on thrombosis is associated with platelet hyperactivity (75). Cancer cells induce activation of platelets by different types of mechanisms; however, the common outcome of this activation is an increase in metastatic spread of cancer cells $(73,76)$. The link between platelets and cancer metastases seems to be a common feature of different types of cancers. Moreover, platelets contribute to the metastatic process in many ways including enhancing tumor cell adhesion in the vasculature, a process where P-selectin seems to have a prominent role $(74,76)$. Indeed, mice deficient of P-selectin had fewer metastases in the lungs than WT mice when injected with colon cancer cells (77). In the same study, it was also shown that cancer cells in the lung vasculature were surrounded by mouse platelets, an event that was greatly reduced in P-selectin ${ }^{-1-}$ mice. Similarly, lung metastases were significantly reduced in P-selectin ${ }^{-/-}$mice in a syngeneic model of metastatic melanoma (78). However, when P-selectin was selectively deleted in platelets, melanoma metastasis were attenuated but not at the same extent as the $\mathrm{KO}$ mice, uncovering an essential role of endothelial P-selectin in lung metastasis. Subsequent studies highlighted that the absence of platelets not only attenuate melanoma metastasis in the lungs but also increase liver metastases, suggesting that platelets may specifically direct metastases in the lungs (66). Organ-specific vasculature anatomy, as well as hemodynamic, may explain in part the different requirement of platelets as they may assist tumor cell attachment in the lungs where the blood flow is naturally fast but are dispensable in the liver, where P-selectin expressed on endothelial cells is sufficient to capture tumor cells due to inherently slow blood stream. Formation of tumor emboli or tumor aggregates may also directly regulate the expression levels of selectin ligands on tumor cells. Cells from the metastatic breast cancer line MDA-MB-231 do not roll on E-selectin in vitro (79). However, when cultured in 3D spheroids, these cells upregulate E-selectin ligands and exhibit robust rolling on E-selectin in vitro. Induction of E-selectin ligands on spheroids may depend on the secretion of pro-inflammatory cytokines, such as interleukin 6 (IL6) and TNF $\alpha$.

\section{Selectin and the Establishment of a Metastatic Niche}

The predisposition of certain organs to support metastases of specific cancer is long recognized and represents the basis of the "seed and soil" hypothesis proposed by Steven Paget more than 100 years ago (80). The generation of a permissive microenvironment or a premetastatic niche to enable circulating cancer cells to colonize distant organs is crucial for an efficient establishment of metastases $(80,81)$. Several factors contribute to the formation of a premetastatic niche, such as primary tumor-derived secreting factors, exosomes, bone marrow-derived cells, and stromal cells. Selectins and their ligands may also contribute to shape the metastatic niche. In a syngeneic mouse model of lung metastases, it has been shown that vascular endothelial growth factor (VEGF) and 
other tumor-derived secreting factors promote focal adhesion kinase- (FAK)-dependent expression of E-selectin in the lung endothelium, thereby facilitating cancer cell homing in the lungs (56). Since selectins are one of the main mediators of leukocytes trafficking, it is conceivable that selectins contribute to leukocyte recruitment at metastatic and premetastatic sites. P- and L-selectin-mediated interactions between tumor cells, platelets, and leukocytes have been shown to induce endothelial activation with concomitant induction of C-C Chemokine Ligand 5 (CCL5) expression in vitro and in vivo (82). Moreover, CCL5 expression could be blocked by an anti-P-selectin blocking antibody demonstrating the importance of selectins in this process. Monocyte homing to metastatic sites was dependent on the expression of CLL5 from the activated endothelium, and remarkably, inhibition of monocyte recruitment greatly decreased metastases by reducing cancer cell survival. Subsequently, it was shown that monocyte recruitment at metastatic site was dependent on monocytic expression of PSGL-1, a well characterized ubiquitously selectin ligand (65). In this study, the MC-38 GFP colon carcinoma cell line showed increased numbers of metastasis in the lungs of C57BL/6 mice compared to the FUT7-deficient mice. Metastases were rescued by injecting selectin ligands bearing leukocytes, highlighting the importance of selectins/selectin ligands interactions in establishing a prometastatic niche.

\section{Selectin and Cancer Cells Retention in the Niche}

It has been demonstrated that E-selectin regulates hematopoietic stem cells (HSC) dormancy in the bone marrow niche (83). Indeed, HSC quiescence and self-renewal were enhanced in E-selectin ${ }^{-/-}$ mice. Although to our knowledge, there is no evidence for such a role of selectins in solid tumors, retention of leukemic cells in their niche might be mediated in part by selectins. An example is represented by AML where E-selectin plays a role in retention and protection of the leukemic stem cells (LSC) in the bone marrow vascular niche (84). Indeed, LCS from an AML mouse model, where E-selectin was knocked out, showed greater sensitivity toward cytarabine compared to WT AML mice. Moreover, treatment with GMI-1271, a small molecule glycomimetic, rationally designed based on the bioactive conformation of SLe ${ }^{a / x}$ and a potent and specific antagonist of E-selectin, caused mobilization of the LCS into the blood, suggesting that E-selectin contributes to the retention of LSC in the bone marrow vasculature niche. Within the bone marrow niche, E-selectin might protect AML cells by regulating key pathways, such as Wnt [Wingless-type mouse mammary tumor virus (MMTV) integration site family member] and sonic Hedgehog (52). E-selectin was shown to regulate cell cycle in LSCs of a murine retroviral transduction/ transplantation model of CML, suggesting that E-selectin may also be an important component of the bone marrow vascular niche in CML (85). In MM, PSGL-1/P-selectin interactions seem to be important not only for homing of malignant cells to the bone marrow but also in mediating the interactions between the malignant cells and the bone marrow niche, where PSGL-1 regulates myeloma proliferation and resistance to therapy (35).

\section{TARGETING THE SELECTINS AND THEIR LIGANDS}

Inhibition of selectin-selectin ligand interactions could impact different aspects of the tumor from metastatic spread to reshaping the metastatic niche. The consequence of this strategy depends certainly on the type of cancer and the stage at diagnosis. Inhibition of selectin/selectin ligand interactions can be achieved by different strategies, including inhibition of key enzymes responsible for generating the selectin ligands, such as STs, or blockage of selectin/selectin ligands interaction (Figure 3); in the latter case, the use of a variety of compounds can be employed spanning from small molecules, glycomimetics, heparin derivatives, and blocking antibodies.

\section{ST Inhibition}

Due to the broad scope of biological processes regulated by sialic acid-containing glycoconjugates in both health and disease, many studies have aimed to develop ST inhibitors (86). While several of these have successfully develop potent inhibitors with partial selectivity against individual family members in vitro, the large majority of the developed compounds are not cell permeable, since they incorporate CMP or a derivative thereof giving the compounds negative charge and not enabling them to cross the cell membrane, let alone into the Golgi apparatus where their targets are located. A breakthrough in a general approach to developing glycosyltransferase inhibitors came when Vocadlo and colleagues introduced a Trojan Horse approach that involves the use of the cells' own metabolic machinery to convert an unnatural monosaccharide into its corresponding nucleotide diphosphate donor substrate (87). Paulson and colleagues subsequently applied this approach to the development of the first cell permeable ST inhibitor described as $3 \mathrm{~F}_{\mathrm{ax}}$-Neu5Ac (88). Delivered to cells in its peracetylated form enables it to readily diffuse across the cell membrane, be deacetylated by cellular esterases and subsequently be formed into CMP- $3 \mathrm{~F}_{\mathrm{ax}}-\mathrm{Neu} 5 \mathrm{Ac}$ (Figure 4), which acts as a very poor substrate for the STs by virtue of the electron withdrawing effects of the fluorine substituent (89). As a consequence, $\mathrm{CMP}-3 \mathrm{~F}_{\mathrm{ax}}-\mathrm{Neu}$ Ac builds up to high levels inside the cells and impairs the actions of all mammalian STs examined to date, both in vitro (88) and in vivo (90).

The therapeutic potential of $3 \mathrm{~F}_{\mathrm{ax}}-\mathrm{Neu} 5 \mathrm{Ac}$ in reversing the hypersialylated state of cancer cells was realized by Adema and colleagues, where they demonstrated that treating skin melanoma with $3 \mathrm{~F}_{\mathrm{ax}}-\mathrm{Neu} 5 \mathrm{Ac}$ prior to injecting cells into the mice greatly impaired tumor growth in vivo (91). These results nicely demonstrated the potential of ST inhibition as a cancer therapeutic. Nevertheless, due to critical roles played by sialylation in healthy tissues, the non-selective nature of this inhibitor is a concern. In particular, an important role for sialylation was confirmed within the kidney in a study where $3 \mathrm{~F}_{\mathrm{ax}}-\mathrm{Neu} 5 \mathrm{Ac}$ was systemically administered to mice (90). At certain doses, $3 \mathrm{~F}_{\mathrm{ax}}-\mathrm{Neu} 5 \mathrm{Ac}$ caused irreversible kidney damage, resulting in loss of protein in within the urine, significant loss of weight, and the eventual death of the animals. A similar phenotype has also been observed in mice deficient in sialic acid biosynthesis (92). It is noteworthy that 


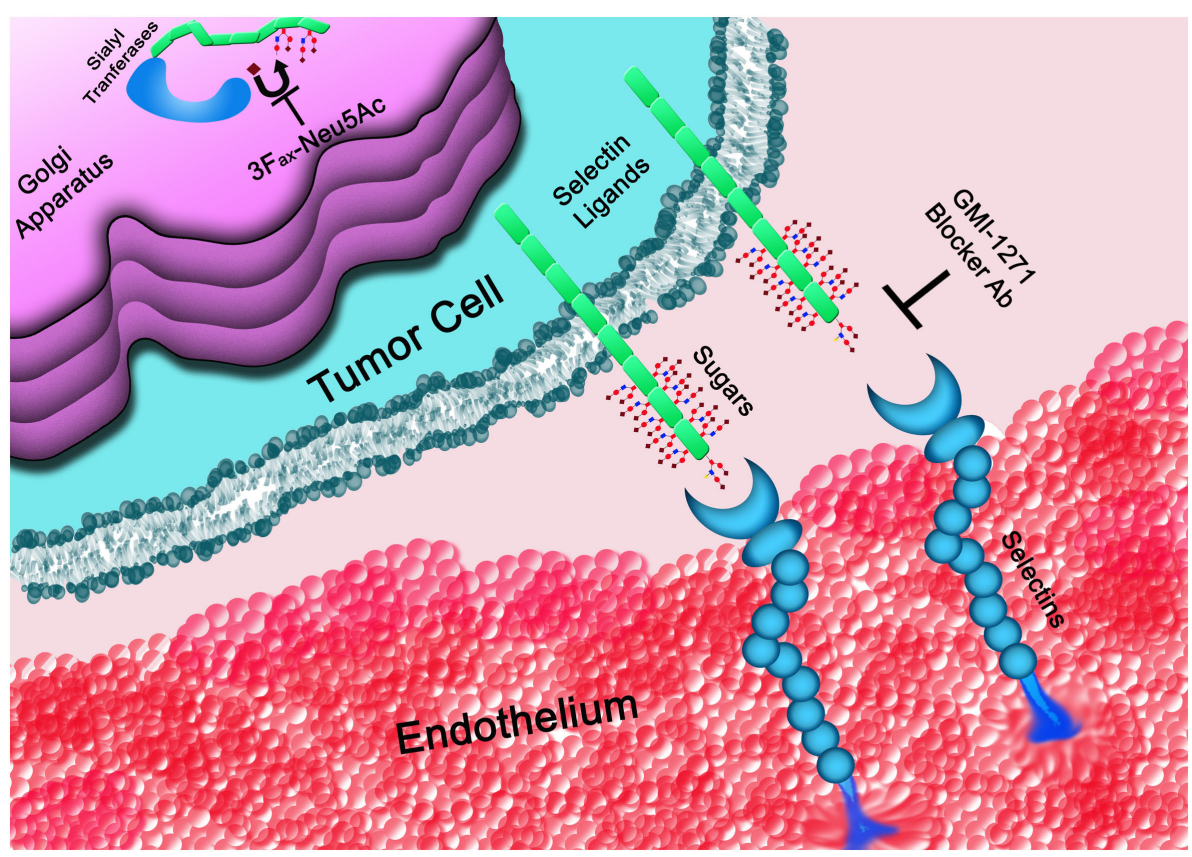

FIGURE 3 | The glycans determinants required for selectins binding are generated within the Golgi apparatus. STs, such as ST3GAL6, are responsible for the covalent linkage of sialic acid ( to the underline sugar backbone. Once on the cell surface, selectin ligands interact with selectins mediating cell rolling on the vasculature endothelium. Interactions of tumor cells with platelets or the endothelium (represented in the figure) can be blocked at the level of the Golgi apparatus by inhibiting STs or by directly interfering with the binding to selectins using blocker antibodies (Ab) or small molecules, such as GMI-1271, among others.

out of the numerous ST-deficient mice established to date, none have developed such a phenotype. Based on these observations, it is likely that multiple STs work together to form the negatively charged glomerular filtration system on podocytes within the kidney. Hence, selectively inhibiting an individual ST in vivo may be feasible without the severe secondary consequences to the host observed with the global ST inhibitor.

A more complete structural knowledge of the ST family may enable selective ST inhibitors to be developed. Without these in hand, however, Adema and colleagues have developed an innovative approach to selectively deliver $3 \mathrm{~F}_{\mathrm{ax}}-\mathrm{Neu} 5 \mathrm{Ac}$ to cells in vivo (22). Working with poly lactic acid (PLGA) nanoparticles to encapsulate $3 \mathrm{~F}_{\mathrm{ax}}$-Neu5Ac, the compound was successfully delivered to melanoma cells by virtue of an antibody displayed on the surface of the nanoparticles that is specific for a tumor antigen. Targeting $3 \mathrm{~F}_{\mathrm{ax}}-\mathrm{Neu}$ Ac to the cancer cells in this way allowed for delivery of the compound to cells within mice, preventing metastasis of the melanoma cells to the lung. This approach appears to have broad applicability for different forms of cancer, so long as a specific tumor antigen is known. While work in this area is still in its infancy, modulating ST activity with small molecule inhibitors is an exciting avenue with broad therapeutic potential.

\section{Small Molecules, Blocking Antibodies, Glycomimetics, and Heparin Derivatives}

Inhibition of P-selectin has been shown to reduce metastasis in vivo in different types of tumor. In MM, PSGL-1 has been shown to regulate the interaction between the malignant cells and their microenvironment, including macrophages $(35,93)$. Moreover, PSGL-1/P-selectin interactions mediate homing of the myeloma cells to the bone marrow and resistance to chemotherapy in the context of the bone marrow niche in vivo. Importantly, inhibition of PSGL-1/P-selectin interactions by the glycomimetic compound GMI-1070, or by humanized blocker antibodies to P-selectin or PSGL-1, was able to suppress homing of myeloma cells to the bone marrow and restore sensitivity to bortezomib $(35,50)$. Recently, holothurian glycosaminoglycan (hGAG), a marine compound similar to heparin sodium, has been shown to decrease melanoma lung metastasis in mice possible through the inhibition of P-selectin although other mechanisms may account for this effect (94). Metastases are attenuated by heparin and low-molecular fraction heparins whose mechanism(s) of action, among others, is the suppression of platelets-tumor cells and endothelial-tumor cells interactions following P-selectin inhibition (95-97). Similar mechanism(s) is shared by other glycan mimetics or molecules, such as sulfated hexasaccharides and ascidian dermatan sulfates $(98,99)$.

Although MM universally express ligands for P-selectin, we have recently shown that rolling on E-selectin is restricted to a minority of myeloma cells expressing functional E-selectin ligands $(51,69)$. Moreover, when xenotransplanted into a SCID mouse model, the E-selectin ligands expressing myeloma cells gave rise to a remarkably aggressive disease characterized by resistance to bortezomib (51). Notably, the glycomimetic E-selectin inhibitor GMI-1271 improved survival and restored 


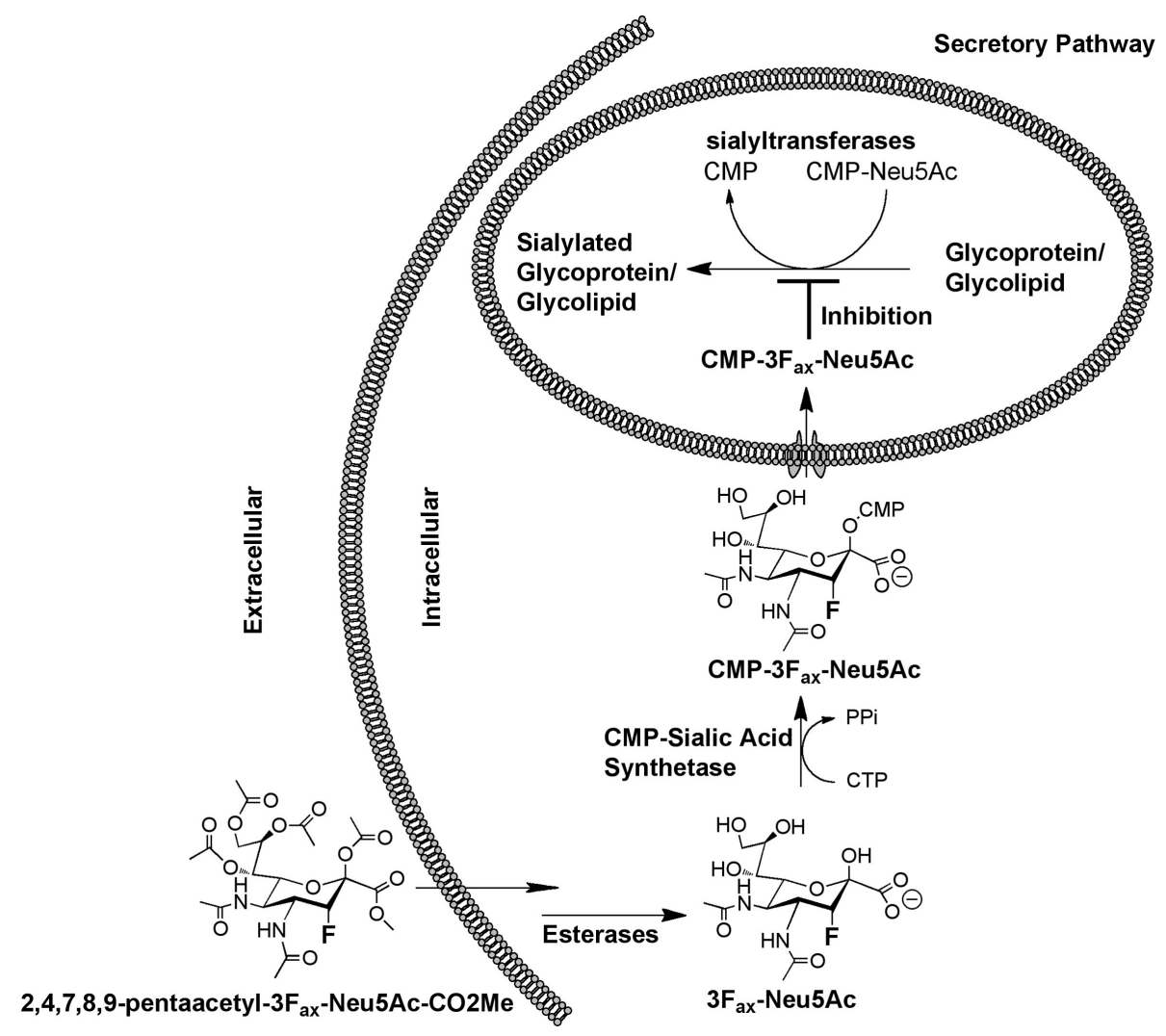

FIGURE 4 | Mechanism of action of the CMP-3F ${ }_{a x}-N e u 5 A c$ inhibitor (see text for details)

the anti-myeloma activity of bortezomib. GMI-1271 was also able to reduce leukemia burden in primary AML blasts engrafted non-obese diabetic (NOD) SCID IL2 receptor $\gamma(\mathrm{c})$ null $\left(\mathrm{R} \gamma \mathrm{c}^{-/-}\right)$ mice when combined with chemotherapy (52). GMI-1271 in combination with standard chemotherapy showed promising results in reducing metastatic lesions in xenograft mouse models of pancreatic and breast cancer $(100,101)$. In the latter, it was also shown that GMI-1271 targets MCF7 breast cancer stem cells $\left(\mathrm{CD} 44^{+} \mathrm{CD} 24^{-}\right)$homing to the bone marrow, pointing out a role of E-selectin in bone marrow dissemination of cancer stem cells in breast cancer (102). A first in human experience with GMI-1271 demonstrated favorable safety together with no mobilization of human stem cells (103). Targeting E-selectin may also be beneficial in alleviating the side effects of chemotherapy and, therefore, improve patient survival and quality of life, especially in elderly and vulnerable patients. In this respect, inhibition of E-selectin by GMI-1271 was shown to alleviate mucositis, resulting from the recruitment of inflammatory macrophages to the damaged intestine after chemotherapy. Indeed, a marked upregulation of E-selectin was detected in the intestine following chemo or radiotherapy, which is critical to the recruitment of inflammatory cells to the mucosa (104).

Based on these results and the promising preclinical findings in AML, a clinical trial to determine the safety, pharmacokinetics, and efficacy of GMI-1271 in combination with chemotherapy in
AML is currently going on (NCT02306291). Moreover, based on the emerging data implicating E-selectin and its ligands in MM, a phase I trial evaluating GMI-1271, as an adjunct to bortezomibbased therapy, is planned. Molecules similar to GMI-1271 have also been developed, in particular GMI-1359, which exhibits dual specificity toward E-selectin and CXCR4. In combination with standard chemotherapy, this molecule showed the ability to reduce metastases in xenograft mouse models of pancreatic and prostate cancers and improved survival in a xenograft mouse model of AML (105-107).

\section{Exploiting Selectin/Selectin Ligand Interactions}

Targeting selectins may also represent an attractive strategy to deliver chemotherapeutic drugs to sites of metastases. Melanoma lung metastases were decreased by an E-selectin-targeted polymer-drug conjugates. In this study, an E-selectin binding peptide was conjugated with $\mathrm{N}$-(2-hydroxylpropyl) methacrylamide and equipped with doxorubicin or a proapoptotic peptide that can be released at mild pH conditions (108). The copolymer effectively decreased melanoma lungs metastases and significantly improved mice survival. As melanoma cancer cells induce the expression of E-selectin in the lung and liver, the copolymers were accumulation primarily in these tissues. A drug-free copolymer 
was also able to prevent lung metastases, suggesting decreased cancer cells extravasation due to inhibition of E-selectin/selectin ligands interactions. Similarly, low-molecular-weight, heparincoated, doxorubicin-loaded liposomes were shown to inhibit melanoma metastases to the lungs by inhibiting tumor cells platelets formations (109).

Leukocytes have been functionalized by selectin-coated liposomes to deliver proapoptotic molecules, such as tumor necrosis factor related apoptosis inducing ligand (TRAIL), to tumor cells. E-selectin-coated liposomes containing TRAIL were used to "equip" circulation leukocytes with TRAIL by taking advantage of the interactions between E-selectin and its ligands expressed on the surface of leukocytes $(110,111)$. The "equipped" leukocytes were able to efficiently clear circulating colon cancer and prostate cancer cells in a xenograft mouse model.

\section{FUTURE PERSPECTIVES}

In conclusion, there is mounting evidence supporting a role for selectins and their ligands in cancer patients, promoting

\section{REFERENCES}

1. Carvalho S, Catarino TA, Dias AM, Kato M, Almeida A, Hessling B, et al. Preventing E-cadherin aberrant N-glycosylation at Asn-554 improves its critical function in gastric cancer. Oncogene (2016) 35:1619-31. doi:10.1038/ onc. 2015.225

2. Stowell SR, Ju T, Cummings RD. Protein glycosylation in cancer. Annu Rev Pathol (2015) 10:473-510. doi:10.1146/annurev-pathol-012414-040438

3. Reymond N, d'Agua BB, Ridley AJ. Crossing the endothelial barrier during metastasis. Nat Rev Cancer (2013) 13:858-70. doi:10.1038/nrc3628

4. Dennis JW, Granovsky M, Warren CE. Glycoprotein glycosylation and cancer progression. Biochim Biophys Acta (1999) 1473:21-34. doi:10.1016/ S0304-4165(99)00167-1

5. Freire-de-Lima L. Sweet and sour: the impact of differential glycosylation in cancer cells undergoing epithelial-mesenchymal transition. Front Oncol (2014) 4:59. doi:10.3389/fonc.2014.00059

6. Hauselmann I, Borsig L. Altered tumor-cell glycosylation promotes metastasis. Front Oncol (2014) 4:28. doi:10.3389/fonc.2014.00028

7. Glavey SV, Huynh D, Reagan MR, Manier S, Moschetta M, Kawano Y, et al. The cancer glycome: carbohydrates as mediators of metastasis. Blood Rev (2015) 29:269-79. doi:10.1016/j.blre.2015.01.003

8. Bull C, Stoel MA, den Brok MH, Adema GJ. Sialic acids sweeten a tumor's life. Cancer Res (2014) 74:3199-204. doi:10.1158/0008-5472.CAN-14-0728

9. Schultz MJ, Swindall AF, Bellis SL. Regulation of the metastatic cell phenotype by sialylated glycans. Cancer Metastasis Rev (2012) 31:501-18. doi:10.1007/ s10555-012-9359-7

10. Harduin-Lepers A, Vallejo-Ruiz V, Krzewinski-Recchi MA, Samyn-Petit B, Julien S, Delannoy P. The human sialyltransferase family. Biochimie (2001) 83:727-37. doi:10.1016/S0300-9084(01)01301-3

11. Datta AK. Comparative sequence analysis in the sialyltransferase protein family: analysis of motifs. Curr Drug Targets (2009) 10:483-98. doi:10.2174/138945009788488422

12. Takashima S, Tsuji S. Functional diversity of mammalian sialyltransferases. Trends Glycosci Glycotechnol (2011) 23:178-93. doi:10.4052/tigg.23.178

13. Cohen M, Varki A. The sialome - far more than the sum of its parts. OMICS (2010) 14:455-64. doi:10.1089/omi.2009.0148

14. Harduin-Lepers A, Mollicone R, Delannoy P, Oriol R. The animal sialyltransferases and sialyltransferase-related genes: a phylogenetic approach. Glycobiology (2005) 15:805-17. doi:10.1093/glycob/cwi063

15. Rao FV, Rich JR, Rakic B, Buddai S, Schwartz MF, Johnson K, et al. Structural insight into mammalian sialyltransferases. Nat Struct Mol Biol (2009) 16:1186-8. doi:10.1038/nsmb. 1685 metastatic behavior and disease progression. The emergence of new therapeutic strategies will hopefully lead to an improvement in outcome of patients with aberrant selectin ligand expression.

\section{AUTHOR CONTRIBUTIONS}

MO contributed to writing the manuscript, communicated with journal editor, and submitted the manuscript. AN and MM contributed to writing the manuscript and preparing the figures.

\section{ACKNOWLEDGMENTS}

We apologize to all colleagues whose important work could not be cited due to space limitations.

\section{FUNDING}

This work was supported by grants from the Health Research Board HRB CSA2012/10, Science Foundation Ireland, and Irish Cancer Society 14/ICS/B3042 (both to MO).

16. Kuhn B, Benz J, Greif M, Engel AM, Sobek H, Rudolph MG. The structure of human alpha-2,6-sialyltransferase reveals the binding mode of complex glycans. Acta Crystallogr D Biol Crystallogr (2013) 69:1826-38. doi:10.1107/ S0907444913015412

17. Meng L, Forouhar F, Thieker D, Gao Z, Ramiah A, Moniz H, et al. Enzymatic basis for N-glycan sialylation: structure of rat alpha2,6-sialyltransferase (ST6GAL1) reveals conserved and unique features for glycan sialylation. J Biol Chem (2013) 288:34680-98. doi:10.1074/jbc.M113.519041

18. Volkers G, Worrall LJ, Kwan DH, Yu CC, Baumann L, Lameignere E, et al. Structure of human ST8SiaIII sialyltransferase provides insight into cell-surface polysialylation. Nat Struct Mol Biol (2015) 22:627-35. doi:10.1038/ nsmb.3060

19. Harduin-Lepers A, Krzewinski-Recchi MA, Colomb F, Foulquier F, GrouxDegroote S, Delannoy P. Sialyltransferases functions in cancers. Front Biosci (Elite Ed) (2012) 4:499-515. doi:10.2741/E396

20. Bull C, den Brok MH, Adema GJ. Sweet escape: sialic acids in tumor immune evasion. Biochim Biophys Acta (2014) 1846:238-46. doi:10.1016/j. bbcan.2014.07.005

21. Dall'Olio F, Malagolini N, Trinchera M, Chiricolo M. Sialosignaling: sialyltransferases as engines of self-fueling loops in cancer progression. Biochim Biophys Acta (2014) 1840:2752-64. doi:10.1016/j.bbagen.2014.06.006

22. Bull C, Boltje TJ, van Dinther EAW, Peters T, de Graaf AMA, Leusen JHW, et al. Targeted delivery of a sialic acid-blocking glycomimetic to cancer cells inhibits metastatic spread. ACS Nano (2015) 9:733-45. doi:10.1021/ nn5061964

23. Vajaria BN, Patel KR, Begum R, Patel PS. Sialylation: an avenue to target cancer cells. Pathol Oncol Res (2015) 1-5. doi:10.1007/s12253-015-0033-6

24. Pearce OM, Laubli H. Sialic acids in cancer biology and immunity. Glycobiology (2016) 26:111-28. doi:10.1093/glycob/cwv097

25. Chong YK, Sandanaraj E, Koh LW, Thangaveloo M, Tan MS, Koh GR, et al. ST3GAL1-associated transcriptomic program in glioblastoma tumor growth, invasion, and prognosis. J Natl Cancer Inst (2015) 108:djv326. doi:10.1093/ jnci/djv326

26. Hedlund M, Ng E, Varki A, Varki NM. Alpha 2-6-Linked sialic acids on $\mathrm{N}$-glycans modulate carcinoma differentiation in vivo. Cancer Res (2008) 68:388-94. doi:10.1158/0008-5472.CAN-07-1340

27. Antony P, Rose M, Heidenreich A, Knuchel R, Gaisa NT, Dahl E. Epigenetic inactivation of ST6GAL1 in human bladder cancer. BMC Cancer (2014) 14:901. doi:10.1186/1471-2407-14-901

28. Lee M, Lee HJ, Bae S, Lee YS. Protein sialylation by sialyltransferase involves radiation resistance. Mol Cancer Res (2008) 6:1316-25. doi:10.1158/15417786.MCR-07-2209 
29. Myers DD, Wrobleski SK, Kelsey K, Farris D, Jose DA, Peter HK, et al. E-selectin inhibitor GMI-1271 works in combination with low-molecular weight heparin to decrease venous thrombosis and bleeding risk in a mouse model. Blood (2014) 124:593.

30. Hudak JE, Canham SM, Bertozzi CR. Glycocalyx engineering reveals a Siglec-based mechanism for NK cell immunoevasion. Nat Chem Biol (2014) 10:69-75. doi:10.1038/nchembio. 1388

31. Jandus C, Boligan KF, Chijioke O, Liu H, Dahlhaus M, Demoulins T, et al. Interactions between Siglec-7/9 receptors and ligands influence NK celldependent tumor immunosurveillance. J Clin Invest (2014) 124:1810-20. doi:10.1172/JCI65899

32. Laubli H, Pearce OM, Schwarz F, Siddiqui SS, Deng L, Stanczak MA, et al. Engagement of myelomonocytic Siglecs by tumor-associated ligands modulates the innate immune response to cancer. Proc Natl Acad Sci U S A (2014) 111:14211-6. doi:10.1073/pnas.1409580111

33. Hamamura K, Furukawa K, Hayashi T, Hattori T, Nakano J, Nakashima H, et al. Ganglioside GD3 promotes cell growth and invasion through p130Cas and paxillin in malignant melanoma cells. Proc Natl Acad Sci U S A (2005) 102:11041-6. doi:10.1073/pnas.0503658102

34. Seales EC, Jurado GA, Brunson BA, Wakefield JK, Frost AR, Beilis SL. Hypersialylation of beta(1) integrins, observed in colon adenocarcinoma, may contribute to cancer progression by up-regulating cell motility. Cancer Res (2005) 65:4645-52. doi:10.1158/0008-5472.CAN-04-3117

35. Azab AK, Quang P, Azab F, Pitsillides C, Thompson B, Chonghaile T, et al. $\mathrm{P}$-selectin glycoprotein ligand regulates the interaction of multiple myeloma cells with the bone marrow microenvironment. Blood (2012) 119:1468-78. doi:10.1182/blood-2011-07-368050

36. Glavey SV, Manier S, Natoni A, Sacco A, Moschetta M, Reagan MR, et al. The sialyltransferase ST3GAL6 influences homing and survival in multiple myeloma. Blood (2014) 124:1765-76. doi:10.1182/ blood-2014-03-560862

37. Julien S, Ivetic A, Grigoriadis A, QiZe D, Burford B, Sproviero D, et al. Selectin ligand sialyl-Lewis $\mathrm{X}$ antigen drives metastasis of hormone-dependent breast cancers. Cancer Res (2011) 71:7683-93. doi:10.1158/0008-5472. CAN-11-1139

38. Tozawa K, Okamoto T, Kawai N, Hashimoto Y, Hayashi Y, Kohri K. Positive correlation between sialyl Lewis $\mathrm{X}$ expression and pathologic findings in renal cell carcinoma. Kidney Int (2005) 67:1391-6. doi:10.1111/j.1523-1755.2005.00216.x

39. Fukuoka K, Narita N, Saijo N. Increased expression of sialyl Lewis(x) antigen is associated with distant metastasis in lung cancer patients: immunohistochemical study on bronchofiberscopic biopsy specimens. Lung Cancer (1998) 20:109-16. doi:10.1016/S0169-5002(98)00016-6

40. Patel KD, Cuvelier SL, Wiehler S. Selectins: critical mediators of leukocyte recruitment. Semin Immunol (2002) 14:73-81. doi:10.1006/ smim.2001.0344

41. Sperandio M. Selectins and glycosyltransferases in leukocyte rolling in vivo. FEBS J (2006) 273:4377-89. doi:10.1111/j.1742-4658.2006.05437.x

42. Gout S, Tremblay PL, Huot J. Selectins and selectin ligands in extravasation of cancer cells and organ selectivity of metastasis. Clin Exp Metastasis (2008) 25:335-44. doi:10.1007/s10585-007-9096-4

43. Witz IP. The selectin-selectin ligand axis in tumor progression. Cancer Metastasis Rev (2008) 27:19-30. doi:10.1007/s10555-007-9101-z

44. Laubli H, Borsig L. Selectins promote tumor metastasis. Semin Cancer Biol (2010) 20:169-77. doi:10.1016/j.semcancer.2010.04.005

45. Bendas G, Borsig L. Cancer cell adhesion and metastasis: selectins, integrins, and the inhibitory potential of heparins. Int J Cell Biol (2012) 2012:676731. doi:10.1155/2012/676731

46. Heidemann F, Schildt A, Schmid K, Bruns OT, Riecken K, Jung C, et al. Selectins mediate small cell lung cancer systemic metastasis. PLoS One (2014) 9:e92327. doi:10.1371/journal.pone.0092327

47. Ben-David T, Sagi-Assif O, Meshel T, Lifshitz V, Yron I, Witz IP. The involvement of the sLe-a selectin ligand in the extravasation of human colorectal carcinoma cells. Immunol Lett (2008) 116:218-24. doi:10.1016/j. imlet.2007.11.022

48. Geng Y, Yeh K, Takatani T, King MR. Three to tango: MUC1 as a ligand for both E-selectin and ICAM-1 in the breast cancer metastatic cascade. Front Oncol (2012) 2:76. doi:10.3389/fonc.2012.00076
49. Li J, Guillebon AD, Hsu JW, Barthel SR, Dimitroff CJ, Lee YF, et al. Human fucosyltransferase 6 enables prostate cancer metastasis to bone. Br J Cancer (2013) 109:3014-22. doi:10.1038/bjc.2013.690

50. Muz B, Azab F, de la Puente P, Rollins S, Alvarez R, Kawar Z, et al. Inhibition of P-Selectin and PSGL-1 using humanized monoclonal antibodies increases the sensitivity of multiple myeloma cells to Bortezomib. Biomed Res Int (2015) 2015:417586. doi:10.1155/2015/417586

51. Natoni A, Smith TAG, Keane N, Locatelli-Hoops SC, Oliva I, Fogler WE, et al. E-selectin ligand expression increases with progression of myeloma and induces drug resistance in a murine transplant model, which is overcome by the glycomimetic E-selectin antagonist, GMI-1271. Blood (2015) 126:1805.

52. Chien S, Haq SU, Pawlus M, Moon RT, Estey EH, Appelbaum FR, et al. Adhesion of acute myeloid leukemia blasts to E-selectin in the vascular niche enhances their survival by mechanisms such as wnt activation. Blood (2013) 122:61.

53. Gakhar G, Navarro VN, Jurish M, Lee GY, Tagawa ST, Akhtar NH, et al. Circulating tumor cells from prostate cancer patients interact with E-selectin under physiologic blood flow. PLoS One (2013) 8:e85143. doi:10.1371/ journal.pone.0085143

54. Biancone L, Araki M, Araki K, Vassalli P, Stamenkovic I. Redirection of tumor metastasis by expression of E-selectin in vivo. J Exp Med (1996) 183:581-7. doi:10.1084/jem.183.2.581

55. Brodt P, Fallavollita L, Bresalier RS, Meterissian S, Norton CR, Wolitzky BA. Liver endothelial E-selectin mediates carcinoma cell adhesion and promotes liver metastasis. Int J Cancer (1997) 71:612-9. doi:10.1002/ (SICI)1097-0215(19970516)71:4<612::AID-IJC17>3.3.CO;2-1

56. Hiratsuka S, Goel S, Kamoun WS, Maru Y, Fukumura D, Duda DG, et al. Endothelial focal adhesion kinase mediates cancer cell homing to discrete regions of the lungs via E-selectin up-regulation. Proc Natl Acad Sci U S A (2011) 108:3725-30. doi:10.1073/pnas.1100446108

57. Khatib AM, Fallavollita L, Wancewicz EV, Monia BP, Brodt P. Inhibition of hepatic endothelial E-selectin expression by C-raf antisense oligonucleotides blocks colorectal carcinoma liver metastasis. Cancer Res (2002) 62:5393-8.

58. Wicklein D, Schmidt A, Labitzky V, Ullrich S, Valent P, Schumacher U. Eand p-selectins are essential for repopulation of chronic myelogenous and chronic eosinophilic leukemias in a scid mouse xenograft model. PLoS One (2013) 8:e70139. doi:10.1371/journal.pone.0070139

59. Krause DS, Lazarides K, Lewis JB, von Andrian UH, Van Etten RA. Selectins and their ligands are required for homing and engraftment of BCR-ABL1+ leukemic stem cells in the bone marrow niche. Blood (2014) 123:1361-71. doi:10.1182/blood-2013-11-538694

60. Lafouresse F, Bellard E, Laurent C, Moussion C, Fournie JJ, Ysebaert L, et al. L-selectin controls trafficking of chronic lymphocytic leukemia cells in lymph node high endothelial venules in vivo. Blood (2015) 126:1336-45. doi:10.1182/blood-2015-02-626291

61. Laubli H, Stevenson JL, Varki A, Varki NM, Borsig L. L-selectin facilitation of metastasis involves temporal induction of Fut7-dependent ligands at sites of tumor cell arrest. Cancer Res (2006) 66:1536-42. doi:10.1158/0008-5472. CAN-05-3121

62. Laubli H, Borsig L. Selectins as mediators of lung metastasis. Cancer Microenviron (2010) 3:97-105. doi:10.1007/s12307-010-0043-6

63. Zollner O, Lenter MC, Blanks JE, Borges E, Steegmaier M, Zerwes HG, et al. L-selectin from human, but not from mouse neutrophils binds directly to E-selectin. J Cell Biol (1997) 136:707-16. doi:10.1083/jcb.136.3.707

64. Jutila MA, Kurk S, Jackiw L, Knibbs RN, Stoolman LM. L-selectin serves as an E-selectin ligand on cultured human T lymphoblasts. J Immunol (2002) 169:1768-73. doi:10.4049/jimmunol.169.4.1768

65. Hoos A, Protsyuk D, Borsig L. Metastatic growth progression caused by PSGL-1-mediated recruitment of monocytes to metastatic sites. Cancer Res (2014) 74:695-704. doi:10.1158/0008-5472.CAN-13-0946

66. Coupland LA, Chong BH, Parish CR. Platelets and P-selectin control tumor cell metastasis in an organ-specific manner and independently of NK cells. Cancer Res (2012) 72:4662-71. doi:10.1158/0008-5472.CAN-11-4010

67. Kannagi R. Molecular mechanism for cancer-associated induction of sialyl Lewis X and sialyl Lewis A expression-the Warburg effect revisited. Glycoconj $J$ (2004) 20:353-64. doi:10.1023/B:GLYC.0000033631.35357.41

68. Koike T, Kimura N, Miyazaki K, Yabuta T, Kumamoto K, Takenoshita S, et al. Hypoxia induces adhesion molecules on cancer cells: a missing link between 
Warburg effect and induction of selectin-ligand carbohydrates. Proc Natl Acad Sci U S A (2004) 101:8132-7. doi:10.1073/pnas.0402088101

69. Natoni A, Moschetta M, Glavey S, Wu P, Morgan GJ, Joshi L, et al. Multiple myeloma cells express functional E-selectin ligands which can be inhibited both in-vitro and in-vivo leading to prolongation of survival in a murine transplant model. Blood (2014) 124:4718.

70. Hakomori S, Kannagi R. Glycosphingolipids as tumor-associated and differentiation markers. J Natl Cancer Inst (1983) 71:231-51.

71. Hakomori S. Tumor-associated glycolipid antigens, their metabolism and organization. Chem Phys Lipids (1986) 42:209-33. doi:10.1016/0009-3084(86)90054-X

72. Sakuma K, Aoki M, Kannagi R. Transcription factors c-Myc and CDX2 mediate E-selectin ligand expression in colon cancer cells undergoing EGF/ bFGF-induced epithelial-mesenchymal transition. Proc Natl Acad Sci U S A (2012) 109:7776-81. doi:10.1073/pnas.1111135109

73. Li N. Platelets in cancer metastasis: to help the "villain" to do evil. Int J Cancer (2016) 138:2078-87. doi:10.1002/ijc.29847

74. Hoffman R, Haim N, Brenner B. Cancer and thrombosis revisited. Blood Rev (2001) 15:61-7. doi:10.1054/blre.2001.0149

75. Cooke NM, Egan K, McFadden S, Grogan L, Breathnach OS, O'Leary J, et al. Increased platelet reactivity in patients with late-stage metastatic cancer. Cancer Med (2013) 2:564-70. doi:10.1002/cam4.86

76. Tesfamariam B. Involvement of platelets in tumor cell metastasis. Pharmacol Ther (2016) 157:112-9. doi:10.1016/j.pharmthera.2015.11.005

77. Kim YJ, Borsig L, Varki NM, Varki A. P-selectin deficiency attenuates tumor growth and metastasis. Proc Natl Acad Sci U S A (1998) 95:9325-30. doi:10.1073/pnas.95.16.9325

78. Ludwig RJ, Boehme B, Podda M, Henschler R, Jager E, Tandi C, et al. Endothelial P-selectin as a target of heparin action in experimental melanoma lung metastasis. Cancer Res (2004) 64:2743-50. doi:10.1158/00085472.CAN-03-1054

79. Geng Y, Chandrasekaran S, Hsu JW, Gidwani M, Hughes AD, King MR. Phenotypic switch in blood: effects of pro-inflammatory cytokines on breast cancer cell aggregation and adhesion. PLoS One (2013) 8:e54959. doi:10.1371/journal.pone.0054959

80. Sceneay J, Smyth MJ, Moller A. The pre-metastatic niche: finding common ground. Cancer Metastasis Rev (2013) 32:449-64. doi:10.1007/ s10555-013-9420-1

81. Smith HA, Kang Y. The metastasis-promoting roles of tumor-associated immune cells. JMol Med (Berl) (2013) 91:411-29. doi:10.1007/ s00109-013-1021-5

82. Laubli H, Spanaus KS, Borsig L. Selectin-mediated activation of endothelial cells induces expression of CCL5 and promotes metastasis through recruitment of monocytes. Blood (2009) 114:4583-91. doi:10.1182/ blood-2008-10-186585

83. Winkler IG, Barbier V, Nowlan B, Jacobsen RN, Forristal CE, Patton JT, et al. Vascular niche E-selectin regulates hematopoietic stem cell dormancy, self renewal and chemoresistance. Nat Med (2012) 18:1651-7. doi:10.1038/ nm. 2969

84. Winkler IG, Barbier V, Pattabiraman DR, Gonda TJ, Magnani JL, Levesque JP. Vascular niche E-selectin protects acute myeloid leukaemia stem cells from chemotherapy. Blood (2014) 124:516.

85. Aggoune D, Magnani JL, Van Etten RA, Krause DS. The vascular niche is involved in regulating leukemic stem cells in murine chronic myelogenous leukemia. Blood (2014) 124:516.

86. Wang L, Liu Y, Wu L, Sun XL. Sialyltransferase inhibition and recent advances. Biochim Biophys Acta (2016) 1864:143-53. doi:10.1016/j. bbapap.2015.07.007

87. Gloster TM, Zandberg WF, Heinonen JE, Shen DL, Deng L, Vocadlo DJ. Hijacking a biosynthetic pathway yields a glycosyltransferase inhibitor within cells. Nat Chem Biol (2011) 7:174-81. doi:10.1038/nchembio.520

88. Rillahan CD, Antonopoulos A, Lefort CT, Sonon R, Azadi P, Ley K, et al. Global metabolic inhibitors of sialyl- and fucosyltransferases remodel the glycome. Nat Chem Biol (2012) 8:661-8. doi:10.1038/nchembio.999

89. Burkart MD, Vincent SP, Duffels A, Murray BW, Ley SV, Wong CH. Chemo-enzymatic synthesis of fluorinated sugar nucleotide: useful mechanistic probes for glycosyltransferases. Bioorg Med Chem (2000) 8:1937-46. doi:10.1016/S0968-0896(00)00139-5
90. Macauley MS, Arlian BM, Rillahan CD, Pang PC, Bortell N, Marcondes $\mathrm{MC}$, et al. Systemic blockade of sialylation in mice with a global inhibitor of sialyltransferases. J Biol Chem (2014) 289:35149-58. doi:10.1074/jbc. M114.606517

91. Bull C, Boltje TJ, Wassink M, de Graaf AMA, van Delft FL, den Brok MH, et al. Targeting aberrant sialylation in cancer cells using a fluorinated sialic acid analog impairs adhesion, migration, and in vivo tumor growth. Mol Cancer Ther (2013) 12:1935-46. doi:10.1158/1535-7163.MCT-13-0279

92. Galeano B, Klootwijk R, Manoli I, Sun M, Ciccone C, Darvish D, et al. Mutation in the key enzyme of sialic acid biosynthesis causes severe glomerular proteinuria and is rescued by $\mathrm{N}$-acetylmannosamine. J Clin Invest (2007) 117:1585-94. doi:10.1172/JCI30954

93. Zheng Y, Yang J, Qian J, Qiu P, Hanabuchi S, Lu Y, et al. PSGL-1/selectin and ICAM-1/CD18 interactions are involved in macrophage-induced drug resistance in myeloma. Leukemia (2013) 27:702-10. doi:10.1038/leu.2012.272

94. Yue Z, Wang A, Zhu Z, Tao L, Li Y, Zhou L, et al. Holothurian glycosaminoglycan inhibits metastasis via inhibition of P-selectin in B16F10 melanoma cells. Mol Cell Biochem (2015) 410:143-54. doi:10.1007/ s11010-015-2546-4

95. Borsig L. Heparin as an inhibitor of cancer progression. Prog Mol Biol Transl Sci (2010) 93:335-49. doi:10.1016/S1877-1173(10)93014-7

96. Borsig L. Antimetastatic activities of heparins and modified heparins. Experimental evidence. Thromb Res (2010) 125(Suppl2):S66-71. doi:10.1016/ S0049-3848(10)70017-7

97. Gomes AM, Kozlowski EO, Borsig L, Teixeira FC, Vlodavsky I, Pavao MS. Antitumor properties of a new non-anticoagulant heparin analog from the mollusk Nodipecten nodosus: effect on P-selectin, heparanase, metastasis and cellular recruitment. Glycobiology (2015) 25:386-93. doi:10.1093/glycob/ cwul19

98. Borsig L, Vlodavsky I, Ishai-Michaeli R, Torri G, Vismara E. Sulfated hexasaccharides attenuate metastasis by inhibition of P-selectin and heparanase. Neoplasia (2011) 13:445-52. doi:10.1593/neo.101734

99. Kozlowski EO, Pavao MS, Borsig L. Ascidian dermatan sulfates attenuate metastasis, inflammation and thrombosis by inhibition of P-selectin. J Thromb Haemost (2011) 9:1807-15. doi:10.1111/j.1538-7836.2011.04401.x

100. Esposito M, Magnani JL, Kang YB. Exploration of a potent E-selectin antagonist (GMI-1271) as a potential novel therapeutic for treating breast cancer metastasis to the bone and lung. Cancer Res (2014) 74:4039. doi:10.1158/1538-7445.AM2014-4039

101. Steele MM, Radhakrishnan P, Magnani JL, Hollingsworth MA. A small molecule glycomimetic antagonist of E-selectin (GMI-1271) prevents pancreatic tumor metastasis and offers a novel treatment for improved efficacy of chemotherapy. Cancer Res (2014) 74:4503. doi:10.1158/1538-7445. AM2014-4503

102. Price T, Burness M, Sivan A, Cheng R, Magnani JL, Sipkins DA. Breast cancer cells metastasize to bone through E-selectin+ vascular gateways. Cancer Res (2014) 74:4831. doi:10.1158/1538-7445.AM2014-4831

103. Devata S, Sood SL, Hemmer MV, Flanner H, Kramer W, Nietubicz C, et al. First in human phase 1 single dose escalation studies of the E-selectin antagonist GMI-1271 show a favorable safety, pharmacokinetic, and biomarker profile. Blood (2015) 126:1004.

104. Winkler IG, Barbier V, Nutt HL, Hasnain SZ, Levesque JP, Magnani JL, et al. Administration of E-selectin antagonist GMI-1271 improves survival after high-dose chemotherapy by alleviating mucositis and accelerating neutrophil recovery. Blood (2013) 122:2266.

105. Gravina GL, Mancini A, Colapietro A, Monache SD, Angelucci A, Calgani $\mathrm{A}$, et al. Dual E-selectin and CXCR4 inhibition reduces tumor growth and increases the sensitivity to docetaxel in experimental bone metastases of prostate cancer. Cancer Res (2015) 75:428. doi:10.1158/1538-7445. AM2015-428

106. Steele MM, Fogler WF, Magnani JL, Hollingsworth MA. A small molecule glycomimetic antagonist of E-selectin and CXCR4 (GMI-1359) prevents pancreatic tumor metastasis and improves chemotherapy. Cancer Res (2015) 75:425. doi:10.1158/1538-7445.AM2015-425

107. Zhang W, Patel N, Fogler WE, Magnani JL, Andreeff M. The dual E-selectin/ CXCR4 inhibitor, GMI-1359, enhances efficacy of anti-leukemia chemotherapy in FLT3-ITD mutated acute myeloid leukemia. Blood (2015) 126:3790. 
108. Shamay Y, Raviv L, Golan M, Voronov E, Apte RN, David A. Inhibition of primary and metastatic tumors in mice by E-selectin-targeted polymer-drug conjugates. J Control Release (2015) 217:102-12. doi:10.1016/j. jconrel.2015.08.029

109. Chen Y, Peng J, Han M, Omar M, Hu D, Ke X, et al. A low-molecular-weight heparin-coated doxorubicin-liposome for the prevention of melanoma metastasis. J Drug Target (2015) 23:335-46. doi:10.3109/1061186X.2014.996760

110. Mitchell MJ, Wayne E, Rana K, Schaffer CB, King MR. TRAIL-coated leukocytes that kill cancer cells in the circulation. Proc Natl Acad Sci U S A (2014) 111:930-5. doi:10.1073/pnas.1316312111

111. Wayne EC, Chandrasekaran S, Mitchell MJ, Chan MF, Lee RE, Schaffer CB, et al. TRAIL-coated leukocytes that prevent the bloodborne metastasis of prostate cancer. J Control Release (2016) 223:215-23. doi:10.1016/j. jconrel.2015.12.048

Conflict of Interest Statement: MO has received research support from GlycoMimetics Inc. AN and MM have no conflict of interest to declare.

Copyright (C) 2016 Natoni, Macauley and O’Dwyer. This is an open-access article distributed under the terms of the Creative Commons Attribution License (CC BY). The use, distribution or reproduction in other forums is permitted, provided the original author(s) or licensor are credited and that the original publication in this journal is cited, in accordance with accepted academic practice. No use, distribution or reproduction is permitted which does not comply with these terms. 